\title{
Direct Evidence of Polycrystalline Silicon Thin Films Formation during Aluminum Induced Crystallization by In-Situ Heating TEM Observation*1
}

\author{
Seiichiro $\mathrm{Ii}^{1}$, Takeshi Hirota ${ }^{2 * 2}$, Kensuke Fujimoto ${ }^{2 * 3}$, Youhei Sugimoto $^{3 * 4}$, Naoki Takata ${ }^{4}$, \\ Ken-ichi Ikeda ${ }^{5}$, Hideharu Nakashima ${ }^{5}$ and Hiroshi Nakashima ${ }^{6}$ \\ ${ }^{1}$ Department of Mechanical Engineering, Faculty of Engineering, Sojo University, Kumamoto 860-0082, Japan \\ ${ }^{2}$ Department of Molecular and Materials Sciences, Interdisciplinary Graduate School of Engineering Sciences, \\ Kyushu University, Kasuga 816-8580, Japan \\ ${ }^{3}$ Department of Applied Science for Electronics and Materials, Interdisciplinary Graduate School of Engineering Sciences, \\ Kyushu University, Kasuga 816-8580, Japan \\ ${ }^{4}$ Department of Metallurgy and Ceramics Science, Graduate School of Science and Technology, \\ Tokyo Institute of Technology, Tokyo 152-8552, Japan \\ ${ }^{5}$ Department of Electrical and Material Sciences, Faculty of Engineering Sciences, Kyushu University, \\ Kasuga 816-8580, Japan \\ ${ }^{6}$ Art, Science and Technology Center for Cooperative Research, Kyushu University, Kasuga 816-8580, Japan
}

The formation behavior of polycrystalline silicon thin films during the aluminum induced crystallization (AIC) process was investigated by scanning transmission electron microscopy (STEM) and in-situ heating transmission electron microscopy (TEM) observations. The STEM observation and electron dispersive X-ray spectroscopy (EDS) analysis of $e x$-situ heat-treated specimen revealed that the a-Si layer and Al layer switched the positions with each other during the heat treatment, resulting the crystallization of the a-Si layer. Furthermore, the in-situ heating TEM observation and EDS analysis of as-deposited specimen revealed the mixed state of $\mathrm{Si}$ and $\mathrm{Al}$ in an a-Si/Al film and the lateral growth of crystalline Si grain during the heating. The mechanism of AIC and switching layers were also discussed from the experimental results and the binary phase diagram of Al-Si system. [doi:10.2320/matertrans.MRA2007312]

(Received December 17, 2007; Accepted January 21, 2008; Published March 5, 2008)

Keywords: aluminum induced crystallization, polycrystalline silicon thin film, transmission electron microscopy, in-situ heating observation, metastable state

\section{Introduction}

Si thin films on glass substrate are widely applied to a variety of electronic devices. Recently, low-temperature polycrystalline silicon (poly-Si) films on inexpensive substrates (non-alkali glass) are expected to be applied in practice owing to requirement for the good device performance and low fabrication cost. The materials are generally fabricated at low temperatures below $873 \mathrm{~K}$. The solid-phase crystallization (SPC) process is one of conventional way for the poly-Si film fabrication by the crystallization of amorphous $\mathrm{Si}(\mathrm{a}-\mathrm{Si})$ film. However, this process has a trade-off relationship between crystallization temperature and time at temperatures below $873 \mathrm{~K} .{ }^{1)}$ Therefore, for achieving the fabrication of poly-Si film on glass substrate at lower temperature, laser annealing crystallization (LAC) and metal induced crystallization (MIC) have been intensively studied as promising candidates to replace the standard SPC. ${ }^{2,3)}$

Al-induced crystallization (AIC) is one of MIC processes using a metal/a-Si bilayer structure, which can induce the crystallization of a-Si for a rather short time of $5.4 \mathrm{ks}$ even at a low temperature of $773 \mathrm{~K}^{4,5)}$ It has been reported that AIC process is effective for decreasing the activation energy of the

\footnotetext{
${ }^{* 1}$ This paper is Originally Published in Japanese in J. of Japan Institute of Metals 71 (2007) 158-163.

${ }^{* 2}$ Graduate Student, Kyushu University. Present address: Mitsubishi Heavy Industries, Ltd., Japan

${ }^{* 3}$ Graduate Student, Kyushu University

${ }^{* 4}$ Graduate Student, Kyushu University. Present address: Seiko Epson Corp., Japan
}

a-Si crystallization due to the Al layer playing an important role of the catalyst for a-Si crystallization. ${ }^{6)}$ The basic process of the AIC process consists of the heating a-Si/Al/substrate to lower temperature than $850 \mathrm{~K}$, which is a eutectic temperature on the Al-Si binary alloy. During this process, Nast et al. have observed an interesting phenomenon of switching each position of the a-Si/Al bilayer on the glass substrate, resulting in the direct fabrication of poly-Si film on the substrate. ${ }^{4)}$ Nast and Hartmann have pointed out that native Al oxide inserted into the a-Si/Al interface plays an essential role in the final crystal quality of poly-Si film. ${ }^{5)}$ The AIC process with a native $\mathrm{Al}$ oxide layer, i.e., the deposition of an a-Si/Al bilayer with breaking the vacuum, is recently focused on several studies of performing the AIC under various conditions in order to prepare the poly-Si having a large grain size at a low temperature. ${ }^{4-11)}$ On the other hand, Kim et al. have reported that a-Si/Al bilayer deposited under vacuum condition switched positions with each other and the switching rate is higher than that of a-Si/Al bilayer including the oxide film on the top of layers. ${ }^{7)}$ Sugimoto et al. have investigated that the effect of heat-treating temperature and film thickness ratio on the quality of poly-Si by crystal orientation analysis using electron back scattering diffraction and transmission electron microscopy (TEM). ${ }^{12)}$ Moreover, Schneider et al. have focused that the nucleation of $\mathrm{Si}$ in the $\mathrm{Al}_{2} \mathrm{O}_{3} / \mathrm{a}-\mathrm{Si} / \mathrm{Al}$ layers during cooling process after heat-treatment and have reported that the controlling of the nucleation of Si was closely related to the controlling of the growth of $\mathrm{Si}$ during the heating process. However, there are no observations of the crystallization process of a-Si in a-Si/Al bilayer. ${ }^{13,14)}$ 


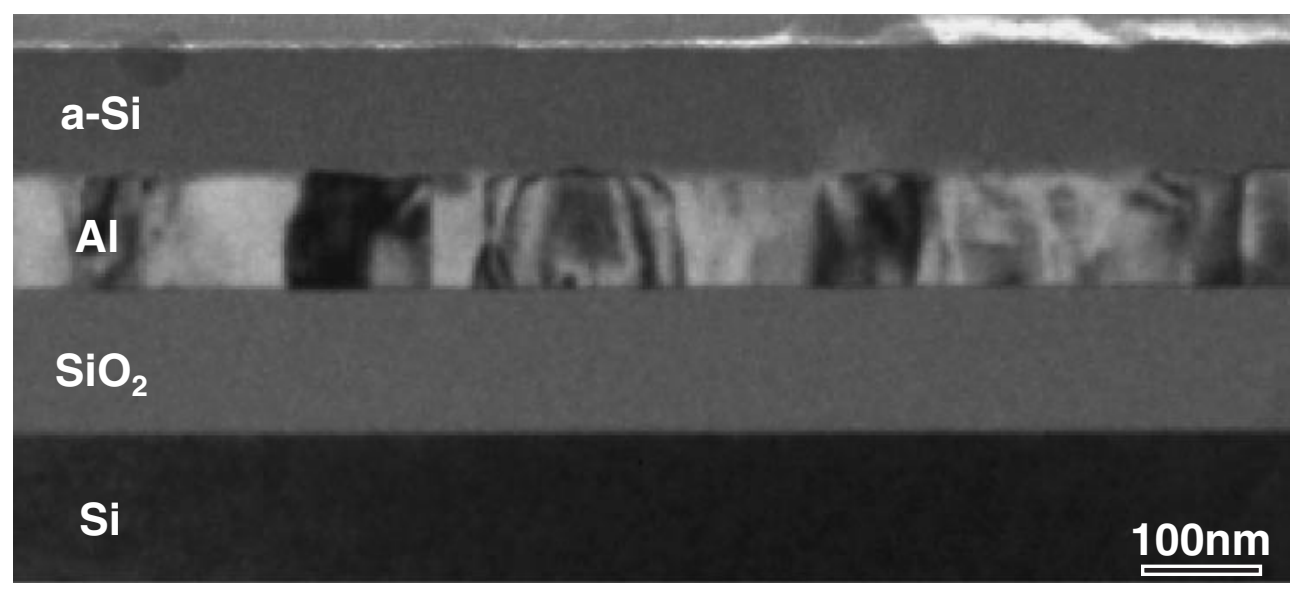

Fig. 1 Cross-sectional BF-TEM image of as-deposited a-Si/Al film.

The purpose of our study is to investigate the microstructural change of a-Si/Al bilayer during heating by using in-situ TEM technique, Scanning TEM (STEM) and energy dispersed X-ray spectroscopy (EDS) systematically. We also discussed crystallizing mechanism from the obtained results.

\section{Experimental Procedure}

$\mathrm{SiO}_{2}$ was thermally grown onto single crystal $\mathrm{Si}$ substrate by dry oxidation at $1273 \mathrm{~K}$ to form the $\mathrm{SiO}_{2} / \mathrm{Si}$ substrate. The thickness of the $\mathrm{SiO}_{2}$ layer was approximately $130 \mathrm{~nm}$. Both $\mathrm{Al}$ and a-Si films were deposited on the $\mathrm{SiO}_{2} / \mathrm{Si}$ substrate by electron beam deposition without breaking the vacuum atmosphere, where both thickness of $\mathrm{Al}$ and a-Si layer were $100 \mathrm{~nm}$. The obtained wafer was annealed at $673 \mathrm{~K}$ for $36 \mathrm{ks}$ in a dry nitrogen atmosphere. The specimen for TEM and STEM observation were prepared by standard microsampling technique using HITACHI FB-2000 focused $\mathrm{Ga}^{+}$ion beam apparatus.

The microstructure and elemental analysis of the $\mathrm{Al} / \mathrm{a}-\mathrm{Si}$ bilayer was performed by TECNAI $20 \mathrm{~F}$ electron microscope equipped with field emission type electron gun and STEM high angle annular dark field (HAADF) image detector. The elemental analysis was measured by energy dispersive X-ray spectroscopy (EDS). In-situ heating TEM observations were also performed by FEI TECNAI 20 and GATAN model 652 double tilt specimen heating holder. The specimens were heated up to 473,553 and $623 \mathrm{~K}$ in the electron microscope. The microstructral change during heating and keeping at those temperatures is recorded by VTR with a flame rate of $30 \mathrm{~s}^{-1}$. In addition, the specimen annealed at $473 \mathrm{~K}$ was kept for $0.3 \mathrm{ks}$ in the microscope, and then each layer was identified by micro area electron diffraction patterns and composition analysis by using EDS measurements. Both electron microscopes were operated at the accelerating voltage of $200 \mathrm{kV}$.

\section{Results and Discussion}

Figure 1 shows a bright field image of the a-Si/Al film as deposited. The observed wafer consists of the $\mathrm{SiO}_{2}, \mathrm{Al}$ and a-Si on the substrate of $\mathrm{Si}$ wafer. We confirmed that the Si substrate of single crystal, amorphous $\mathrm{SiO}_{2}$ and polycrystalline $\mathrm{Al}$ by selected area electron diffraction patterns. Figure 2(a) shows the STEM-HAADF image of the wafer heated at $673 \mathrm{~K}$ for $36 \mathrm{ks}$. And Figs. 2(b) to (d) show elemental maps obtained by EDS measurements. Figure 2 shows that the $\mathrm{Al}$ and a-Si are completely change their positions each other. Sugimoto et al. have reported that the thickness of the crystallized poly-Si layer was closely related to an initial thickness of the Al layer from the cross-sectional microstructure observations of the wafer, and they have concluded that the initial thickness ratio of $\mathrm{Al}$ and a-Si layers was $1: 1$ for the formation of the high quality polycrystalline Si film. ${ }^{11)}$ Therefore, it can be considered that the Si layer shown in Fig. 2 is a part of poly-Si.

In order to observe the microstructural change during heating in direct, we performed the in-situ TEM observation. Figure 3 shows representative images showing of microstructural change of as deposited a-Si/Al bilayer during in-situ heating TEM observation. The temperature is reached up to $533 \mathrm{~K}$. A time interval from Figure 3(a) to (f) is approximately $15 \mathrm{~s}$, respectively. In these figures, the black contrast can be observed in the vicinity of the interface between $\mathrm{Al}$ and a-Si and grows in the a-Si layer. In addition, we confirmed that the further heating enhances the growth of the black contrast to the lateral direction in the initial a-Si layer.

Figure 4(a) and (b) show bright field images of the specimen before (a) and after (b) in-situ heating at $473 \mathrm{k}$ for $0.3 \mathrm{ks}$ in TEM, respectively. It should be noted that the two images were taken from the same region in the specimen. The switching their position of two a-Si and Al layers is starting in the wafer, and the microstructural change was confirmed in the a-Si layer. In addition, it was also observed that the microstructure of the Al layer changed during the insitu heating.

In order to investigate the microstructure of the films after the in-situ heating in detail, we identified the layer by the selected area electron diffraction pattern and STEM-EDS measurement. Figure 5 shows a bright field image (a) in the wafer in-situ heated at $473 \mathrm{~K}$ for $0.3 \mathrm{ks}$ and selected are electron diffraction patterns ((b) to (e)) take from the region $\mathrm{B}$ to $\mathrm{E}$ shown in Fig. 5(a), respectively. Several reflection 


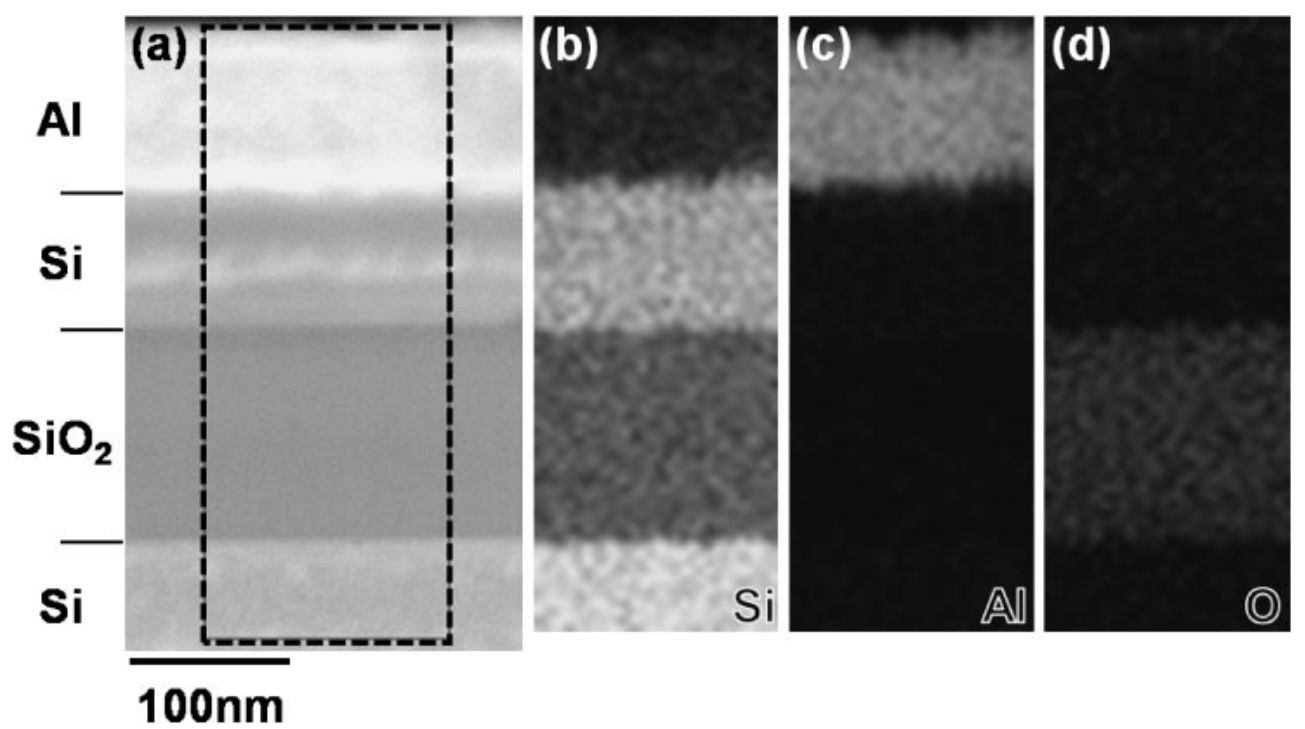

Fig. 2 (a) Cross-sectional HAADF-STEM images of a-Si/Al annealed at $673 \mathrm{~K}$ for $36 \mathrm{ks}$ and elemental maps of (b) Si, (c) $\mathrm{Al}$ and (d) $\mathrm{O}$ of square region in (a).

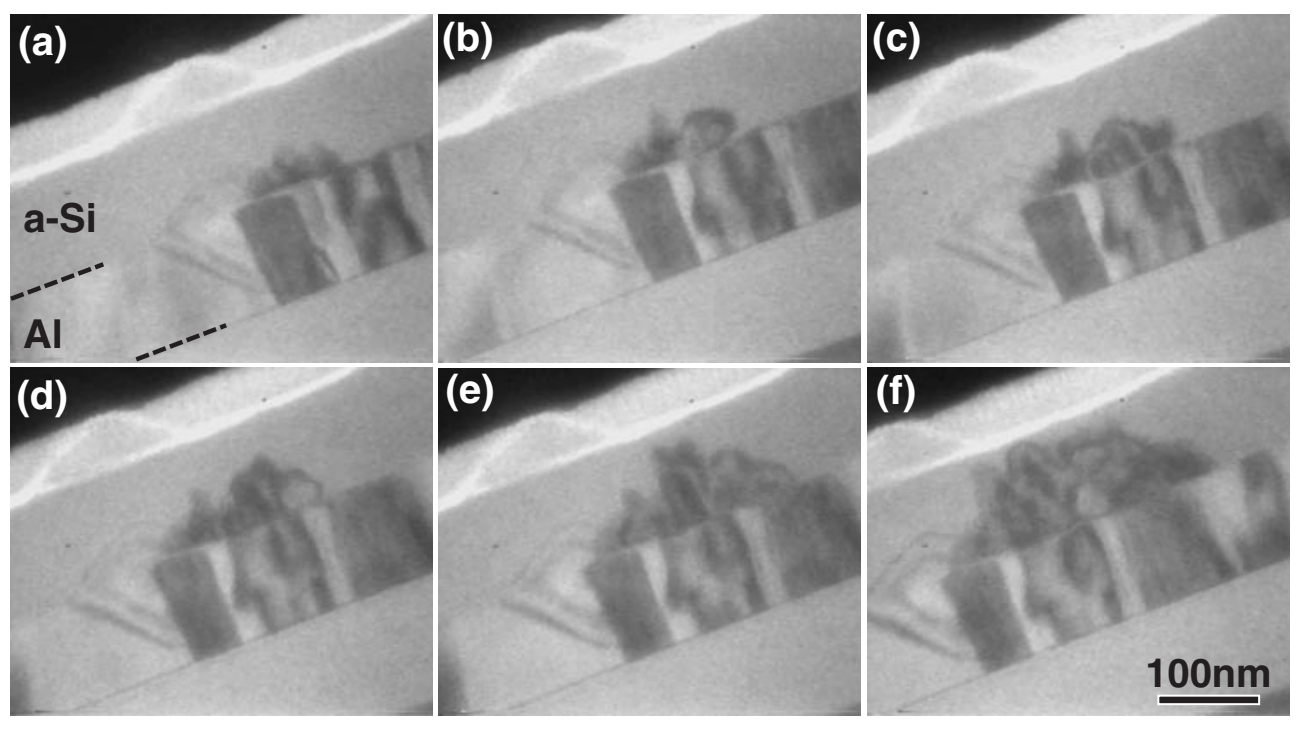

Fig. 3 A series of BF-TEM images of a-Si/Al film taken during in-situ heating at $553 \mathrm{~K}$ (average time interval $15 \mathrm{~s}$ ).

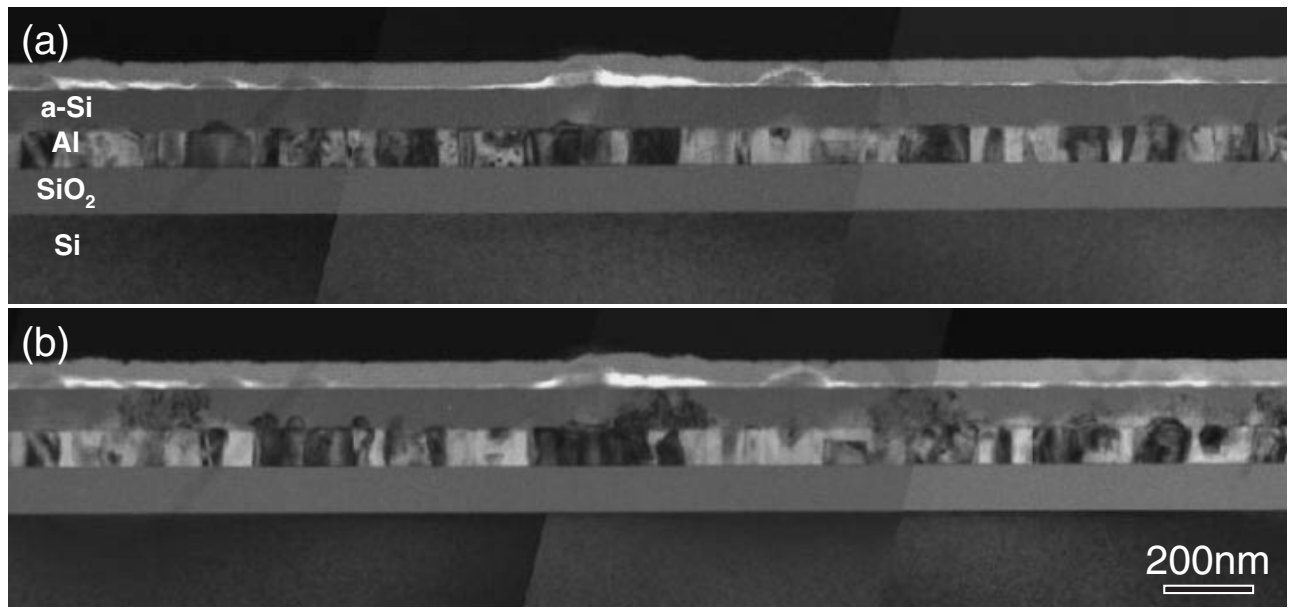

Fig. 4 BF-TEM images of a-Si/Al film. (a) as-deposited and (b) in-situ heated at $473 \mathrm{~K}$ for $0.3 \mathrm{ks}$. 

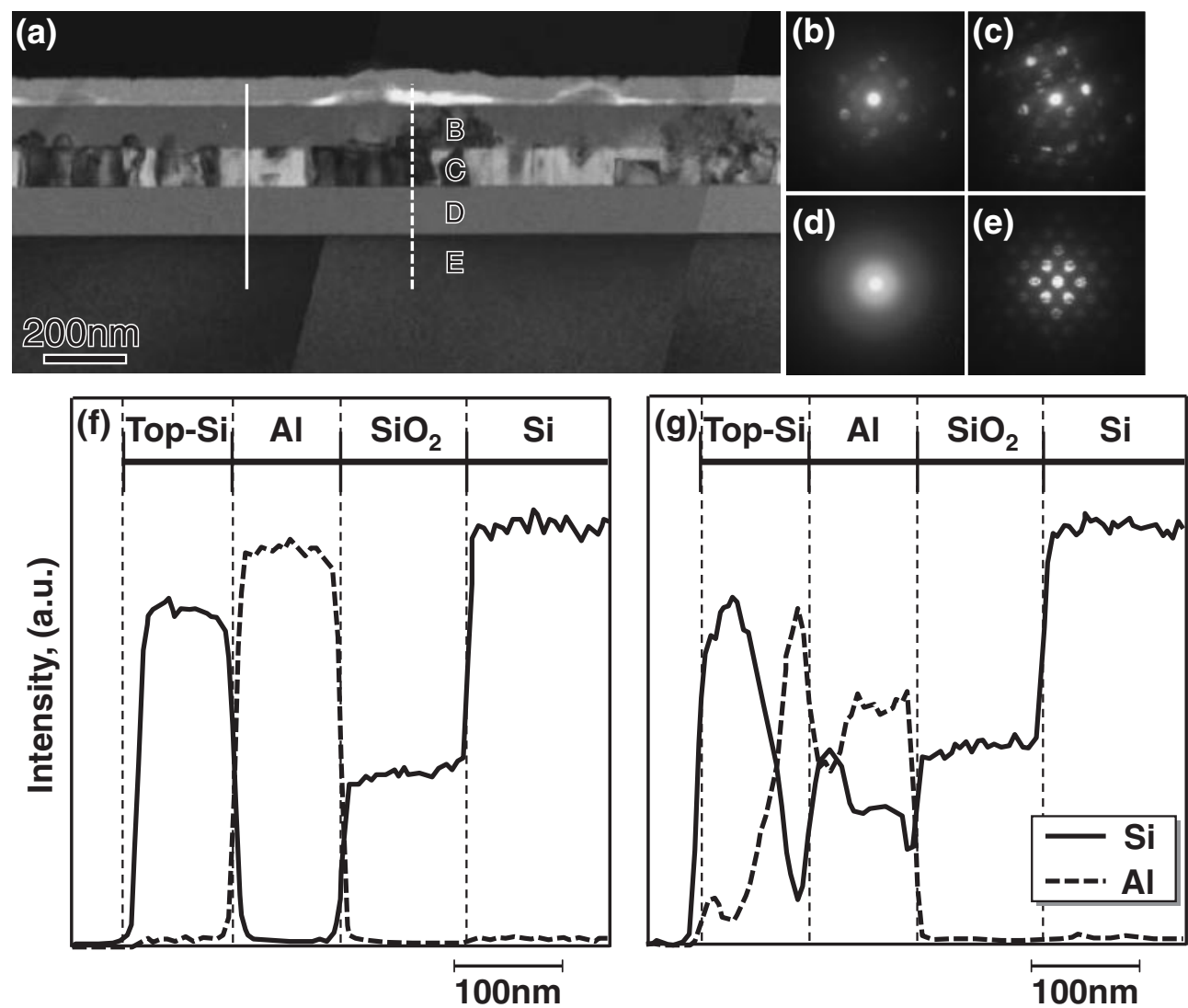

Fig. 5 (a) BF-TEM image, (b) to (e) micro diffraction patterns and (f) and (g) EDS line analysis profile of in-situ heated (at $473 \mathrm{~K}$ for $0.3 \mathrm{ks}) \mathrm{a}-\mathrm{Si} / \mathrm{Al}$ film.
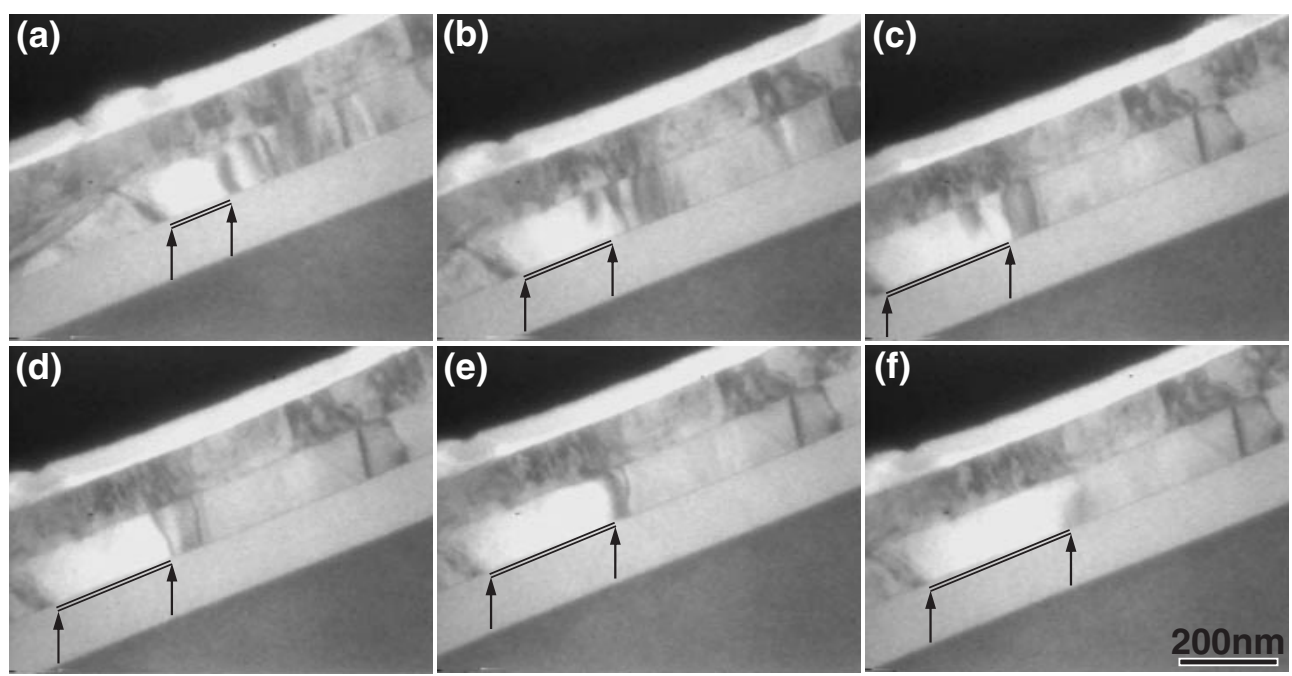

Fig. 6 A series of BF-TEM images of a-Si/Al film taken during in-situ heating at $623 \mathrm{~K}$ (average time interval $15 \mathrm{~s}$ ).

spots obtained from the initial a-Si layer as shown in Fig. 5(b) clearly indicates that the crystallization partially occurs in the a-Si layer during the heating. In addition, we measured the chemical composition of each layers by STEMEDS measurements. Figure 5(f) shows a composition profiles of $\mathrm{Si}$ and $\mathrm{Al}$ measured along white solid line shown in Fig. 5(a). From the Fig. 5(f), we can confirm that the each layer consists of $\mathrm{Si}$ and $\mathrm{Al}$, respectively. On the other hand, the line profiles of other area shown by white broken line in
Fig. 5(a), indicate that $\mathrm{Si}$ and $\mathrm{Al}$ are solved to each other and are mixed state in the $\mathrm{Si}$ and $\mathrm{Al}$ layers. These results suggest that the $\mathrm{Si}$ and $\mathrm{Al}$ layer forms each solid-solution through the interdiffusion at the initial stage of heating, and then $\mathrm{Si}$ is crystallized and forms polycrystalline.

Figure 6 shows representative bright field images of the same specimen shown in Fig. 3 during further heating in TEM up to $623 \mathrm{~K}$. Average time interval shown in Figs. 6(a) to (f) is also $15 \mathrm{~s}$, respectively. The white contrast shown by 


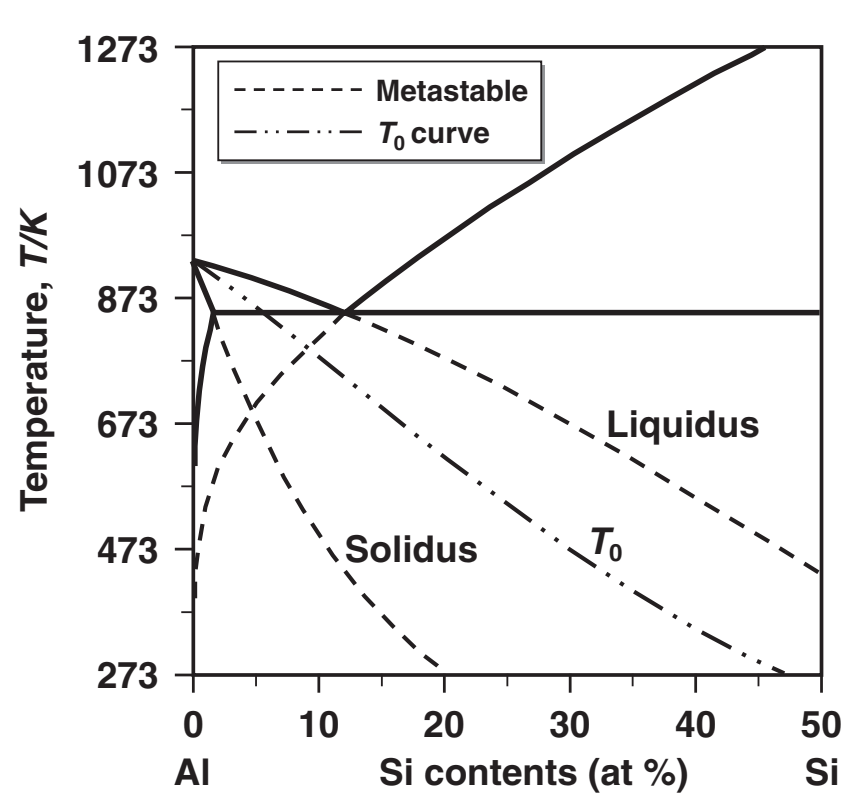

Fig. 7 The Al-Si phase diagram with the metastable line and the $T_{0}$ curve.

double black lines and single black arrows is grown to the lateral direction in the $\mathrm{Al}$ film. Obtained results of the elemental analysis show that the white contrast in the Al layer corresponds to the crystalline $\mathrm{Si}$. Further annealing, we confirmed that the crystalline Si grew to the lateral direction in the polycrystalline $\mathrm{Al}$ layer.

From the obtained results, we considered that the a-Si are crystallized to poly-Si after forming the solid-solution of $\mathrm{Al}$ and $\mathrm{Si}$ in the initial a-Si and $\mathrm{Al}$ layer at early stage of the heating. In addition, crystalline $\mathrm{Si}$ grew to the lateral direction in the Al layer with further heating. In contrast, the Al-Si binary equilibrium phase diagram shown by solid line in Fig. ${ }^{15)}$ represents that Si cannot solve in Al between the temperature between $473 \mathrm{~K}$ and $623 \mathrm{~K}$, which is performed in-situ heating TEM observation. We considered that the specimen investigated in this study is non-equilibrium state, since the film is consisting of amorphous $\mathrm{Si}$ and thin $\mathrm{Al}$ layer on the wafer. A broken line extended from the equilibrium liquidus and solidus line shown in Fig. 7 is the metastable line of $\mathrm{Al}$ in $\mathrm{Al}-\mathrm{Si}$ binary system. In the region between broken liquidus and solidus line, $\mathrm{Si}$ can be solved into $\mathrm{Al}$ even at low temperature region. Especially, the initial Si layer on the substrates exhibits amorphous phase in our studied specimen. Furthermore, both a-Si and Al layers have extremely thin, whose thickness of about $100 \mathrm{~nm}$. These conditions are quite different from that of bulk crystalline materials, that is, equilibrium state, following the equilibrium phase diagram. Therefore, $\mathrm{Si}$ can be solved into the $\mathrm{Al}$ between the 473 and $553 \mathrm{~K}$ as shown in Fig. 7, although this is the non-equilibriums state. That is the reason why the solid solution can be formed during the in-situ heating. Additionally, taking into account metastable line of $\mathrm{Si}$ as the same of $\mathrm{Al}$ as mentioned above, $\mathrm{Si}$ is also solved into the initial $\mathrm{Al}$ layer. However, the specimen is reached to the equilibrium state by further heating, $\mathrm{Al}$ and $\mathrm{Si}$ cannot solved to each other, the mixed $\mathrm{Al}$ and $\mathrm{Si}$ separates into each crystalline phase showing a characteristic morphology of dendritic phase. ${ }^{11)}$
The obtained results suggests that the deposited a-Si and $\mathrm{Al}$ is formed each solid solution because of their nonequilibrium state, and the transition to the equilibrium state grows the crystalline $\mathrm{Si}$. This phenomenon is considered with the same as multilayer consisted of the a-Si and $\mathrm{Al}$ reported by Konno et al. ${ }^{16)}$ Further study about the nucleation site of crystallization is required for understanding the switching their positions of $\mathrm{Si}$ and $\mathrm{Al}$ layers in detail.

\section{Concluding Remarks}

We investigated the microstructural change of a-Si/Al film deposited onto the thermally oxidized Si substrate by using in-situ heating TEM observation and STEM-EDS measurements. The obtained results are shown as follows:

(1) STEM-EDS analysis revealed that the $\mathrm{Al}$ and a-Si layers have switched their positions with each other during the heating at $673 \mathrm{~K}$ for $36 \mathrm{ks}$ in the AIC process.

(2) In-situ heating TEM observation directly revealed the solid solution of $\mathrm{Al}$ in $\mathrm{Si}$ and $\mathrm{Si}$ in $\mathrm{Al}$ and crystallization of $\mathrm{Si}$ during annealing at $473 \mathrm{~K}$. And the crystalline Si grew to the lateral direction in the Al layer with further annealing.

(3) Since the a-Si and Al layers in the wafer used in this study is non-equilibrium state, respectively, $\mathrm{Si}$ and $\mathrm{Al}$ is formed solid-solution to each other in the layers. However, further annealing brings to the equilibrium, therefore $\mathrm{Al}$ and $\mathrm{Si}$ is separated to two layers.

\section{Acknowledgements}

A part of the study is financially supported by Grant-in-Aid for Scientific Research, Ministry of Education, Culture, Sports, Science and Technology, Japan.

\section{REFERENCES}

1) A. T. Voutsas: Appl. Surf. Sci. 208-209 (2003) 250-262.

2) A. Hara, K. Yoshino, F. Takeuchi and N. Sasaki: Jpn. J. Appl. Phys. 42 (2003) 23-27.

3) Z. Jin, G. A. Bhat, M. Yeung, H. S. Kwok and M. Wong: J. Appl. Phys. 84 (1998) 194-200.

4) O. Nast, T. Puzzer, L. M. Koschier, A. B. Sproul and S. R. Wenham: Appl. Phys. Lett. 73 (1998) 3214-3216.

5) S. Gall, M. Muske, I. Sieber, O. Nast and W. Fuhs: J. Non-Cryst. Solids 299-302 (2002) 741-745.

6) M. Doi, F. Katsuki, H. Kumagai and M. Takagi: J. JILM. 57 (2007) 3036. (in Japanese)

7) H. Kim, D. Kim, G. Lee, D. Kim and S. H. Lee: Sol. Energy Mater. Sol. Cells 74 (2002) 323-329.

8) O. Nast and A. J. Hartmann: J. Appl. Phys. 88 (2000) 716-724.

9) I. Sieber, R. Schneider, I. Doerfel, P. Schubert-Bischoff, S. Gall and W. Fuhs: Thin Solid Films 427 (2003) 298-302.

10) O. Nast and S. R. Wenham: J. Appl. Phys. 88 (2000) 124-132.

11) P. I. Widenborg and A. G. Aberle: J. Cryst. Growth 242 (2002) 270282.

12) Y. Sugimoto, N. Takata, T. Hirota, K. Ikeda, F. Yoshida, H. Nakashima and H. Nakashima: Jpn. J. Appl. Phys. 44 (2005) 4770-4775.

13) J. Schneider, A. Schneider, A. Sarikov, J. Klein, M. Muske, S. Gall and W. Fuhs: J. Non-Cryst. Solids 352 (2006) 972-975.

14) A. Sarikov, J. Schneider, M. Muske, S. Gall and W. Fuhs: J. Non-Cryst. Solids 352 (2006) 980-983.

15) T. B. Massalski: Binary Alloy Phase Diagrams Vol. 1, (American Society for Metals) pp. 164-165.

16) T. J. Konno and R. Sinclair: Philos. Mag. B 66 (1992) 749-765. 